\title{
NEW PRECISION ORBITS OF BRIGHT DOUBLE-LINED SPECTROSCOPIC BINARIES. IX. HD 54371, HR 2692, AND 16 URSA MAJORIS
}

\author{
Francis C. Fekel ${ }^{1,5}$, Michael H. Williamson ${ }^{1}$, Matthew W. Muterspaugh ${ }^{1}$, \\ Dimitri Pourbaix ${ }^{2}$, Daryl Willmarth ${ }^{3}$, AND Jocelyn Tomkin ${ }^{4}$ \\ ${ }^{1}$ Center of Excellence in Information Systems, Tennessee State University, 3500 John A. Merritt Boulevard, Box 9501, Nashville, TN 37209, USA; \\ fekel@evans.tsuniv.edu, michael.h.williamson@gmail.com, matthew1@coe.tsuniv.edu \\ ${ }^{2}$ FNRS Institut d'Astronomie et d'Astrophysique, Université Libre de Bruxelles, CP 226, B-1050 Bruxelles, Belgium; pourbaix@astro.ulb.ac.be \\ ${ }^{3}$ Kitt Peak National Obsevatory, P.O. Box 26732, Tucson, AZ 85726-6732, USA; dwillmarth@ noao.edu \\ ${ }^{4}$ Astronomy Department and McDonald Observatory, University of Texas, Austin, TX 78712, USA \\ Received 2014 September 29; accepted 2014 November 30; published 2015 January 19
}

\begin{abstract}
With extensive sets of new radial velocities we have determined orbital elements for three previously known spectroscopic binaries, HD 54371, HR 2692, and 16 UMa. All three systems have had the lines of their secondaries detected for the first time. The orbital periods range from 16.24 to 113.23 days, and the three binaries have modestly or moderately eccentric orbits. The secondary to primary mass ratios range from 0.50 to 0.64 . The orbital dimensions $\left(a_{1} \sin i\right.$ and $\left.a_{2} \sin i\right)$ and minimum masses $\left(m_{1} \sin ^{3} i\right.$ and $\left.m_{2} \sin ^{3} i\right)$ of the binary components all have accuracies of $\leqslant 1 \%$. With our spectroscopic results and the Hipparcos data, we also have determined astrometric orbits for two of the three systems, HR 2692 and 16 UMa. The primaries of HD 54371 and 16 UMa are solar-type stars, and their secondaries are likely K or M dwarfs. The primary of HR 2692 is a late-type subgiant and its secondary is a $\mathrm{G}$ or K dwarf. The primaries of both HR 2692 and 16 UMa may be pseudosynchronously rotating, while that of HD 54371 is rotating faster than its pseudosynchronous velocity.
\end{abstract}

Key words: binaries: spectroscopic - stars: fundamental parameters - stars: individual (HD 54371, HR 2692, 16 UMa) - stars: late-type

Supporting material: machine-readable and VO tables

\section{INTRODUCTION}

In recent decades the intersection of the visual and spectroscopic binary domains has expanded significantly, primarily the result of advances in near-infrared and optical interferometers (Quirrenbach 2001). The analysis of astrometric and spectroscopic observations can produce a complete three-dimensional binary orbit, enabling precise masses and the distance to the system to be determined. Torres et al. (2010) summarized the recent situation, noting that there are more than 20 interferometric binaries with mass uncertainties $\leqslant 3 \%$. Additional basic parameters determined from astrometric and spectroscopic data, such as absolute magnitudes, luminosities, and rotational velocities, enable valuable comparisons with stellar evolutionary theory (e.g., Hummel et al. 2001; Boden et al. 2006; Fekel et al. 2009a).

Fekel \& Tomkin (2004) discussed the initial steps in the current project. In summary, they searched the Eighth Catalogue of the Orbital Elements of Spectroscopic Binary Systems (Batten et al. 1989) for bright, field binaries that could be resolved with current interferometers but had orbits that needed improved precision. While most systems to be reobserved were double-lined binaries, Fekel \& Tomkin (2004) also identified a number of single-lined systems with relatively large mass functions. The hope was that with new red-wavelength high signal-to-noise ratio $(\mathrm{S} / \mathrm{N})$ spectra, lines of the much fainter secondaries could be detected in at least some of the systems. Such an approach has been successful in

\footnotetext{
${ }^{5}$ Visiting Astronomer, Kitt Peak National Observatory, National Optical Astronomy Observatory, operated by the Association of Universities for Research in Astronomy, Inc. under cooperative agreement with the National Science Foundation.
}

the past at finding the secondary spectral features of singlelined main-sequence binaries (e.g., Stockton \& Fekel 1992; Goldberg et al. 2002; Mazeh et al. 2002, 2003; Iliev et al. 2004).

The eight previous papers of our series, the most recent being Fekel et al. (2013b), have produced new and more precise orbits for 23 binaries. Our new spectroscopic orbits are resolvable with the current generation of optical and infrared interferometers such as CHARA on Mount Wilson and NPOI at Lowell Observatory. Our orbits generally produce much more precise minimum masses than previous orbits, so that the spectroscopic results will not be the limiting factor when combined with the astrometry.

Building on the work of Stockton \& Fekel (1992), we have acquired high S/N CCD spectra in the visual region, for three previously known single-lined spectroscopic binaries, HD 54371, HR 2692, and 16 UMa. We have detected their secondary lines and measured their radial velocities, turning those systems into more useful double-lined spectrocopic binaries. The binary mass ratio distribution is an important diagnostic for assessing models of binary formation (e.g., Halbwachs et al. 2003). Some basic data about the three spectroscopic binaries analyzed in this paper are listed in Table 1.

\section{BRIEF HISTORY}

\section{1. $H D 54371=H I P 34567$}

From 22 spectrograms acquired at Mount Wilson Observatory, Sanford (1922) determined HD 54371 $\left(\alpha=07^{\mathrm{h}} 09^{\mathrm{m}} 35^{\mathrm{s}} 39, \delta=25^{\circ} 43^{\prime} 43^{\prime \prime} \cdot 1(2000)\right)$ to be a singlelined spectroscopic binary with an orbital period of 32.8092 
Table 1

Basic Properties of the Program Stars

\begin{tabular}{|c|c|c|c|c|c|c|c|}
\hline Name & HR & HD & Spectral Type ${ }^{a}$ & $\begin{array}{c}V^{\mathrm{b}} \\
(\mathrm{mag})\end{array}$ & $\begin{array}{c}B-V^{\mathrm{b}} \\
(\mathrm{mag})\end{array}$ & $\begin{array}{c}\text { Parallax }^{\mathrm{c}} \\
\text { (mas) }\end{array}$ & $\begin{array}{l}\text { Period } \\
\text { (days) }\end{array}$ \\
\hline$\cdots$ & $\cdots$ & 54371 & G6 V & 7.09 & 0.700 & 39.73 & 32.81 \\
\hline$\cdots$ & 2692 & 54563 & G8 IV & 6.43 & 0.880 & 21.82 & 113.23 \\
\hline $16 \mathrm{UMa}$ & 3648 & 79028 & G0 V & 5.18 & 0.605 & 51.10 & 16.24 \\
\hline
\end{tabular}

a This work

b Perryman \& ESA (1997).

c van Leeuwen (2007).

days and a small eccentricity of 0.08 . With the same radial velocities Lucy \& Sweeney (1971) reexamined the orbit and concluded that it was circular. At Fick Observatory, Beavers \& Salzer (1985) obtained a series of 22 new observations. They combined their radial velocities with those of Sanford (1922) and slightly revised the orbital period to 32.8066 days. They then computed a circular orbit with their velocities alone and stated that their result was a modest improvement over that of Sanford (1922). Abt \& Willmarth (2006) collected seven additional radial velocities at Kitt Peak National Observatory (KPNO) but made only very slight revisions to the orbit of Beavers \& Salzer (1985) and retained the circular solution.

Sanford (1922) estimated a G5 spectral class for HD 54371, while Harlan (1969) and Abt (1985) provided full spectral classifications of G8 V and G5 V, respectively. Gray et al. (2003) gave a similar result of G6 V and concluded that the star has solar abundances.

Strassmeier et al. (2000) examined over 1000 late-type stars, looking for chromospherically active stars that might be amenable to the Doppler imaging technique for star spot reconstruction. They found HD 54371 to have modest $\mathrm{Ca}$ II $\mathrm{H}$ and $\mathrm{K}$ emission, indicating that it is an active star, but its $v$ $\sin i$ value of $5.6 \pm 2.0 \mathrm{~km} \mathrm{~s}^{-1}$ is too low to permit useful Doppler imaging. Mishenina et al. (2008) obtained highresolution spectra of lower main-sequence stars to determine their fundamental parameters and abundances. For HD 54371 they found essentially solar abundances and a $v$ sin $i$ value of $4.9 \pm 1.0 \mathrm{~km} \mathrm{~s}^{-1}$. Observed as part of the very extensive Geneva-Copenhagen Survey of solar neighborhood stars, Holmberg et al. (2009) determined a near solar iron abundance and estimated an age of $10.6 \mathrm{Gyr}$ although with a very large uncertainty. Nakajima \& Morino (2012) considered HD 54371 for membership in the stellar kinematic group IC 2391 but concluded that it was a separate field star.

\section{2. $H R 2692=H D 54563=H I P 34608$}

Reported to have a variable velocity in the fourth edition of The Bright Star Catalogue (Hoffleit \& Jaschek 1982) but without an orbital determination, Beavers \& Salzer (1985) obtained 34 radial-velocity observations of HR 2692 $\left(\alpha=07^{\mathrm{h}} 10^{\mathrm{m}} 06^{\mathrm{s}} .68, \delta=21^{\circ} 14^{\prime} 49^{\prime \prime} .1 \quad(2000)\right)$ at Fick Observatory. Their orbital period of 113.346 days relied on two old velocities from Mount Wilson Observatory (Abt 1970). Adopting many of the orbital elements of Beavers \& Salzer (1985), the Hipparcos team (Perryman \& ESA 1997) computed an astrometric orbit for HR 2692, which was revised by Jancart et al. (2005). Recently, HR 2692 was one of 37 single-lined binaries for which Katoh et al. (2013) obtained very precise radial velocities. From their observations alone, they found an orbital period of 113.2314 days.

Cowley et al. (1967) classified the spectrum of HR 2692 as G9 V, while Harlan (1969) found a similar result, G8 V. Despite these classifications, Beavers \& Salzer (1985) argued that its parallax indicated that the star is likely a subgiant. Massarotti et al. (2008) included HR 2692 in a radial and rotational velocity survey of 761 nearby giants and estimated a rotational velocity of $0.0 \pm 1.0 \mathrm{~km} \mathrm{~s}^{-1}$.

\section{3. $16 U M a=H R 3648=H D 79028=H I P 45333=$ BDS 4962 A}

The binary system $16 \mathrm{UMa}\left(\alpha=09^{\mathrm{h}} 14^{\mathrm{m}} 20.54, \delta=61^{\circ} 25^{\prime}\right.$ $23 ! 9(2000))$ is a close solar neighbor, being just $19.6 \mathrm{pc}$ from the Sun (van Leeuwen 2007). It was one of the first 100 spectroscopic binary systems discovered at the Dominion Astrophysical Observatory (DAO) (Plaskett et al. 1920). Shortly thereafter, based on 24 DAO radial velocities, Young (1923) computed a single-lined spectroscopic orbit that had a period of 16.238 days and a modest eccentricity of 0.09 . Reanalyses of those velocities by Luyten (1936) and Lucy \& Sweeney (1971) indicate that the orbit is indeed eccentric. Abt \& Levy (1976) acquired additional observations at KPNO, combined their velocities with those from the DAO, and obtained new orbital elements that were quite similar to those of Young (1923). As they did with HR 2692, Katoh et al. (2013) obtained numerous high-dispersion echelle spectra of 16 UMa and determined a very precise orbit for it.

Both Cowley (1976) and Cowley \& Bidelman (1979) classified the system as F9 V, while Gray et al. (2003) and Abt (2009) determined classifications of G0 IV-V and G0 V, respectively. Thus, the spectral classifications are in good agreement. Spectroscopic abundance analyses by Edvardssen et al. (1993), Chen et al. (2000), and Takeda et al. (2005) indicate that $16 \mathrm{UMa}$ has a near solar iron abundance.

Being a bright, nearby, solar-type star has resulted in 16 UMa being a part of a variety of chromospheric activity surveys (Wilson \& Skumanich 1964; Young \& Koniges 1977; Hall et al. 2007; Schröder et al. 2009; Martinez-Arnaiz et al. 2010), but no evidence of $\mathrm{Ca}$ II $\mathrm{H}$ and $\mathrm{K}$ emission has been found. Measurements of its $v \sin i$ have resulted in values of $6.1 \mathrm{~km} \mathrm{~s}^{-1}$ (Schröder et al. 2009) and $5.59 \mathrm{~km} \mathrm{~s}^{-1}$ (MartinezArnaiz et al. 2010).

Gomez \& Abt (1982) included 16 UMa in a search at visual wavelengths for the secondaries of single-lined binaries but were unable to detect its secondary. Duquennoy \& Mayor (1991) made 16 UMa part of a large study of stellar multiplicity in solar-type stars and noted that a secondary was not detected in their Coravel observations. Katoh et al. 
Table 2

Telescope, Spectrograph, and Detector Combinations

\begin{tabular}{|c|c|c|c|c|c|}
\hline Telescope & Grating & $\begin{array}{c}\text { CCD } \\
\text { Detector }\end{array}$ & $\begin{array}{c}\text { Central } \\
\text { Wavelength } \\
(\AA)\end{array}$ & $\begin{array}{c}\text { Wavelength } \\
\text { Range } \\
(\AA)\end{array}$ & $\begin{array}{c}\text { Resolving } \\
\text { Power }\end{array}$ \\
\hline Fairborn $2 \mathrm{~m}$ & echelle & SITe ST-002 A & 6010 & 2180 & 35000 \\
\hline Fairborn $2 \mathrm{~m}$ & echelle & Fairchild 486 & 6030 & 4460 & 25000 \\
\hline KPNO coudé feed & $5, \mathrm{~A}^{\mathrm{a}}$ & $\mathrm{F} 3 \mathrm{~KB}$ & 6428 & 5200 & 24000 \\
\hline KPNO coudé feed & $5, \mathrm{~A}^{\mathrm{a}}$ & TI5 & 4536 & 6430 & 30000 \\
\hline McDonald $2.1 \mathrm{~m}$ & echelle & Reticon & 6350 & 1300 & 49000 \\
\hline
\end{tabular}

a Camera 5, grating A.

(2013) also did not find secondary features in their recent highresolution spectra.

\section{OBSERVATIONS AND RADIAL VELOCITIES}

We obtained our observations of the three program stars at three observatories. Most of our spectrograms were acquired from 2003 through 2014 at Fairborn Observatory near Washington Camp in southeast Arizona with the Tennessee State University $2 \mathrm{~m}$ automatic spectroscopic telescope (AST) and fiber-fed echelle spectrograph (Eaton \& Williamson 2004, 2007). Initially, the detector was a $2048 \times 4096$ SITe ST-002A CCD with $15 \mu \mathrm{m}$ pixels. Eaton \& Williamson (2007) have explained the reduction of the raw spectra and their wavelength calibration. Those AST echelle spectrograms have 21 orders that span the wavelength region 4920-7100 A and have an average resolution of $0.17 \AA$ or a resolving power of 35,000 at $6000 \AA$. The $\mathrm{S} / \mathrm{N}$ of these observations at $6000 \AA$ generally ranged from 40 to 60 .

The SITe CCD and its dewar were removed from the AST in the summer of 2011. They were replaced by a Fairchild 486 CCD having a $4096 \times 4096$ array of $15 \mu \mathrm{m}$ pixels and a new dewar (Fekel et al. 2013a). The new echelle spectrograms have 48 orders that range from 3800 to $8260 \AA$. We also installed a $200 \mu \mathrm{m}$ diameter fiber that produces a resolution of $0.24 \AA$ or a resolving power of 25,000 at $6000 \AA$ for the new echelle spectra. Observations with the new detector, dewar, and fiber resulted in much improved $\mathrm{S} / \mathrm{N}$ that, depending on sky conditions, ranged from 100 to 200 at $6000 \AA$.

Spanning the years 2000-2004, Abt \& Willmarth (2006) acquired additional spectrograms of the three binaries at KPNO as part of a survey of solar-type stars that examined their binary frequency and mass ratio distribution. They obtained those observations with the $0.9 \mathrm{~m}$ coudé feed telescope, coudé spectrograph, and a CCD detector identified as F3KB. The spectra covered $320 \AA$, centered on $5200 \AA$, and had a resolution of $0.11 \AA$ pixel $^{-1}$ or a 2 pixel resolving power of 24,000 . The $\mathrm{S} / \mathrm{N}$ of the spectra ranged from 40 to 80 . A more extensive discussion is given in Abt \& Willmarth (2006).

In 2002 and 2003 we collected several spectrograms at McDonald Observatory with the $2.1 \mathrm{~m}$ telescope, the Sandiford Cassegrain echelle spectrograph (McCarthy et al. 1993), and a Reticon CCD. The wavelength region covered by those spectra ranges from 5700 to $7000 \AA$. They have a resolution of $0.13 \AA$ that corresponds to a resolving power of 49,000 at $6350 \AA$. At that central wavelength, the $\mathrm{S} / \mathrm{N}$ of the spectra are $\sim 200$.

Finally, from 2003 through 2007 we acquired several additional spectra at KPNO with the coudé feed telescope, coudé spectrograph, and a Texas Instruments CCD detector.
Those spectra are centered at $6430 \AA$, cover a wavelength range of $84 \AA$, and have a resolution of $0.21 \AA$ or a resolving power of just over 30,000 . They have $\mathrm{S} / \mathrm{N}$ of $\sim 150$. The various telescope, spectrograph, and detector conbinations are summarized in Table 2.

Fekel et al. (2009b) provided an extensive general description of velocity measurement of the Fairborn AST spectra. In summary we measured radial velocities of 168 mostly neutral Fe lines from a solar-type star list that covers the 4920-7120 A region. A Gaussian function was used to fit the features of the narrow-lined stars and determine their radial velocities. The secondary lines were not obvious in the initial measurements of our individual spectra. Thus, to extend our search for those lines, we subtracted the spectrum of the primary, which was obtained by averaging our spectra, appropriately shifted so that all the primary lines from spectrum to spectrum were aligned. This subtraction resulted in a very weak average summed profile of the 168 lines in the residual spectra corresponding to the secondary component. Not all of the residual AST spectra produced detectable features of the secondary. In particular, for HD 54371 and $16 \mathrm{UMa}$ the secondary could only be found in the residual spectra obtained after the upgrades to the telescope system resulted in significantly improved S/N.

The Fairborn velocities are absolute rather than relative velocities. Our unpublished measurements of several IAU standard solar-type stars indicate that the Fairborn Observatory velocities taken with the SITe CCD have a small zero-point offset of $-0.3 \mathrm{~km} \mathrm{~s}^{-1}$ relative to the velocities of Scarfe et al. (1990). Starting in the fall of 2011, velocities from spectra obtained with the new CCD system have a zero-point offset $-0.6 \mathrm{~km} \mathrm{~s}^{-1}$ relative to those of Scarfe et al. (1990). Thus, we added either 0.3 or $0.6 \mathrm{~km} \mathrm{~s}^{-1}$, depending on which detector was used, to each measured velocity.

Abt \& Willmarth (2006) used a cross correlation technique to obtain their KPNO radial velocities. Their reference spectrum was a solar spectrum taken of the daytime sky. Velocity standards came from 64 stars whose velocities appeared to be constant from the survey of Nidever et al. (2002). Abt \& Willmarth (2006) have provided additional information.

Tomkin \& Fekel (2006) have discussed in detail the procedures that we have used to measure the McDonald radial velocities. Like the Fairborn velocities, the McDonald velocities are on an absolute scale.

The KPNO TI CCD spectra, one or two per star, were not measured for radial velocity partly because of their very limited number but also because they do not extend the baseline of the 
observations. However, they were used to determine rotational velocities and spectral types.

\section{DETERMINATION OF SPECTROSCOPIC ORBITS AND RESULTS}

We initially determined the orbital elements of each component with the computer program SB1 (Barker et al. 1967), which iterates the elements with differential corrections. We then used a slightly modified version of that program, called SB2, to obtain a simultaneous solution of both binary components.

To extend the temporal baseline of our observations, in our analyses we have included radial velocities of the three systems that were previously obtained by Abt \& Willmarth (2006). All the velocities for $16 \mathrm{UMa}$ and HD 54371 were published in that paper (Abt \& Willmarth 2006). The radial velocities for HR 2692 , which were also obtained during their survey, are listed here for the first time.

Given the very large magnitude difference between the binary components, which results in the extreme weakness of the secondary features, the primary and secondary velocities of each system clearly have very different velocity precisions. Velocities obtained at different observatories with different spectrographs and detectors can also produce different velocity precisions. Therefore, if the velocities are numerous enough and provide reasonable phase coverage, we have determined individual orbital solutions for the separate data sets of the components from each observatory. The variances of those individual solutions are inversely proportional to the weights that we assigned to the velocities in those different sets.

For two of the three binaries that we analyze below, Katoh et al. (2013) have recently determined very precise orbital elements from their new radial velocities. We have chosen not to combine our observations with theirs, partly because their velocities do not extend the time baseline, but also because Griffin (2013) has raised concerns about some of their work and mentioned that Katoh et al. (2013) appear to have adopted random velocity zero points for their observations. Thus, we have proceeded to make independent orbital solutions and then compare our results with theirs. Although their velocities, as indicated by their rms values, are up to one order of magnitude more precise than ours, the resulting uncertainties of their orbital elements are only about a factor of two better.

We note that the symbols used for the orbital elements, $P, T$, $e, \omega, K$, and $\gamma$ have their customary meanings of orbital period, time of periastron, orbital eccentricity, longitude of periastron, velocity semiamplitude, and center-of-mass velocity, respectively. The physical constants used to determine the related parameters $a \sin i$, the projected orbital separation, and $m \sin ^{3} i$, the minimum mass, which are computed from the orbital elements, are the ones recommended by Torres et al. (2010).

\subsection{HD 54371}

From 2004 through 2014 we acquired 193 spectra of HD 54371 at Fairborn Observatory (Table 3). Additionally, two observations from McDonald Observatory and the seven acquired at KPNO (Abt \& Willmarth 2006) are also listed in Table 3. In some of our Fairborn spectra, we are able to detect the very weak secondary. Figure 1 shows the average profile of the components, summed over 168 regions in a single Fairborn spectrum. After the average primary spectrum was subtracted, the extremely weak secondary is just visible in the residual summed spectrum.

The velocities from McDonald and KPNO are not numerous enough for separate orbital solutions. However, from past experience (e.g., Fekel et al. 2013b) the McDonald and Fairborn velocities have the same zero point. As shown below for $16 \mathrm{UMa}$ and also for HR 2692, the velocities determined by Abt \& Willmarth (2006) also have a very similar zero point and so have not been adjusted.

With the velocities from McDonald, KPNO, and Fairborn given equal weights, we first determined an orbital solution for the primary of HD 54371. The period from that orbit was held constant, and a solution for the velocities of the extremely weak-lined secondary was computed. From those solutions we determined weights of 1.0 for the primary velocities and 0.01 for those of the secondary. A simultaneous solution of the appropriately weighted velocites resulted in the orbital elements and related parameters given in Table 4. The velocities are compared with the computed velocity curves in Figure 2. The high density of points along the velocity curve, especially for the primary, makes it difficult to see the quality of the fit. Thus, in Figure 3 we plot the velocity residuals to the computed curves in separate panels for the primary and secondary.

As is seen in Table 4, our orbit is a substantial improvement over that of Beavers \& Salzer (1985) with the uncertainties of the elements being reduced by a factor of 10 or more in our solution. Although Beavers \& Salzer (1985) and others have adopted a circular orbit, the orbit actually has a modest eccentricity of $0.0708 \pm 0.0006$. Our eccentricity is similar to that initially found by Sanford (1922), which although very uncertain, is $0.08 \pm 0.06$.

\section{2. $H R 2692$}

Our 245 Fairborn Observatory spectra of HR 2692 were acquired between 2003 and 2014 (Table 5). Fourteen additional KPNO observations, obtained between 2000 and 2002 and presented here for the first time, plus two McDonald spectra from 2002 (Table 5) extend the time baseline. In many of our Fairborn spectra, the secondary is weakly visible. Figure 4 shows the average profile of the components summed over 168 regions in a single Fairborn spectrum. After the average primary spectrum was subtracted, the extremely weak secondary is just visible in the residual summed spectrum.

Because of the very large number of Fairborn radial velocities that are well distributed in phase, we initially computed an orbit for the primary of HR 2692 with those velocities. We then determined an orbit with the 14 KPNO radial velocities and found that the center-of-mass velocities for those two orbits are in excellent agreement, differing by less than $0.1 \mathrm{~km} \mathrm{~s}^{-1}$. All the primary velocities from Fairborn, KPNO, and McDonald were given unit weight and a new solution obtained. Then orbital elements for the extremely weak-lined secondary were determined from the Fairborn velocities. Values for the period as well as the shape and orientation of the orbit were taken from the solution of the primary, and only the semiamplitude and center-of-mass velocity were varied. Given the difficulty of measuring the secondary lines, the center-of-mass velocities of the primary and secondary are in reasonable agreement, differing by $0.8 \mathrm{~km} \mathrm{~s}^{-1}$. From the combined primary and secondary velocity solutions we assigned weights of 1.0 to all the primary 


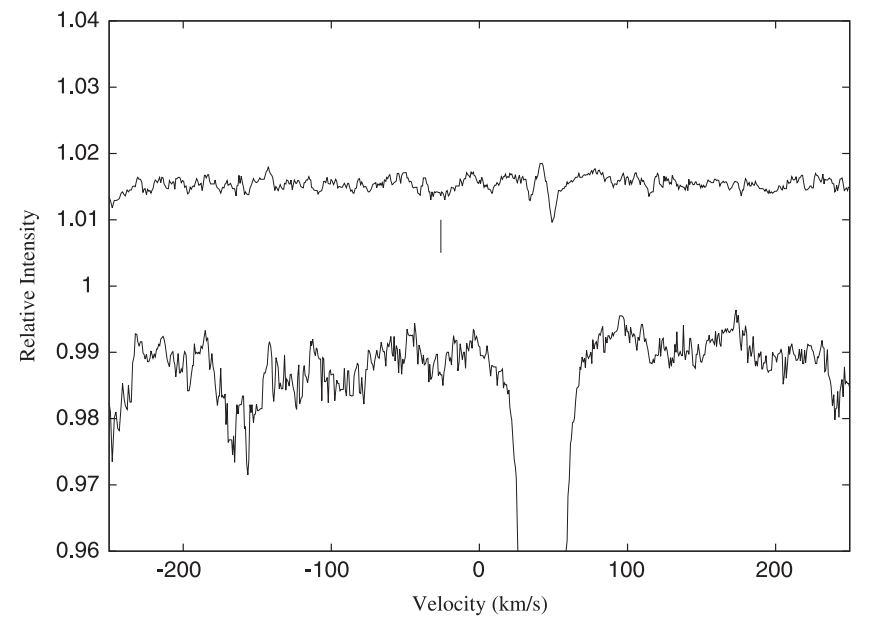

Figure 1. From a Fairborn Observatory spectrum of HD 54371, the lower solid line is the average profile of the components summed over 168 spectral regions. The upper line, arbitrarily vertically shifted for visibility, is the remainder after the average region around the primary component from all useful Fairborn spectra has been removed from the lower line. The position of the secondary is indicated with a tick mark below the summed residual spectrum.
Table 4

Orbital Elements and Related Parameters of HD 54371

\begin{tabular}{lcc}
\hline \hline Parameter & Beavers \& Salzer $(1985)$ & This Study \\
\hline$P$ (days) & $32.8066 \pm 0.0005$ & $32.807302 \pm 0.000078$ \\
$T$ (HJD) & $\ldots$ & $2454663.017 \pm 0.039$ \\
$T_{0}^{\mathrm{a}}(\mathrm{HJD})$ & $2444389.23 \pm 0.22$ & $\ldots$ \\
$e$ & $0.0($ adopted $)$ & $0.07081 \pm 0.00057$ \\
$\omega_{1}(\mathrm{deg})$ & $\ldots$ & $55.09 \pm 0.44$ \\
$K_{1}\left(\mathrm{~km} \mathrm{~s}^{-1}\right)$ & $26.67 \pm 1.33$ & $25.574 \pm 0.014$ \\
$K_{2}\left(\mathrm{~km} \mathrm{~s}^{-1}\right)$ & $\ldots$ & $50.87 \pm 0.23$ \\
$\gamma\left(\mathrm{km} \mathrm{s}^{-1}\right)$ & $19.79 \pm 0.87$ & $20.080 \pm 0.010$ \\
$m_{1} \sin ^{3} i\left(M_{\odot}\right)$ & $\ldots$ & $1.0029 \pm 0.0099$ \\
$m_{2} \sin ^{3} i\left(M_{\odot}\right)$ & $\ldots$ & $0.5042 \pm 0.0029$ \\
$a_{1} \sin i\left(10^{6} \mathrm{~km}^{-}\right)$ & $12.0 \pm 0.6$ & $11.5083 \pm 0.0062$ \\
$a_{2} \sin i\left(10^{6} \mathrm{~km}^{2}\right.$ & $\ldots$ & $22.89 \pm 0.10$ \\
$\mathrm{RV} \mathrm{rms}_{1}\left(\mathrm{~km} \mathrm{~s}^{-1}\right)$ & $\ldots$ & 0.14 \\
$\mathrm{RV} \mathrm{rms}_{2}\left(\mathrm{~km} \mathrm{~s}^{-1}\right)$ & $\ldots$ & 1.59 \\
\hline & &
\end{tabular}

${ }^{a}$ Time of maximum velocity.

Table 3

Radial Velocities of HD 54371

\begin{tabular}{|c|c|c|c|c|c|c|c|c|}
\hline $\begin{array}{l}\text { Hel. Julian Date } \\
\text { (HJD-2400000) }\end{array}$ & Phase & $\begin{array}{c}V_{1} \\
\left(\mathrm{~km} \mathrm{~s}^{-1}\right)\end{array}$ & $\begin{array}{c}(O-C)_{1} \\
\left(\mathrm{~km} \mathrm{~s}^{-1}\right)\end{array}$ & $\mathrm{Wt}_{1}$ & $\begin{array}{c}V_{2} \\
\left(\mathrm{~km} \mathrm{~s}^{-1}\right)\end{array}$ & $\begin{array}{c}(O-C)_{2} \\
\left(\mathrm{~km} \mathrm{~s}^{-1}\right)\end{array}$ & $\mathrm{Wt}_{2}$ & Source \\
\hline 52566.967 & 0.110 & 16.5 & 0.0 & 1.0 & $\ldots$ & $\ldots$ & $\ldots$ & $\mathrm{McD}$ \\
\hline 52719.730 & 0.767 & 41.6 & 0.1 & 1.0 & $\ldots$ & $\ldots$ & $\ldots$ & $\mathrm{McD}$ \\
\hline 53000.843 & 0.335 & -4.4 & 0.0 & 1.0 & $\ldots$ & $\ldots$ & $\ldots$ & KPNO \\
\hline 53001.864 & 0.366 & -3.8 & 0.0 & 1.0 & $\cdots$ & $\cdots$ & $\cdots$ & KPNO \\
\hline 53002.853 & 0.397 & -2.4 & 0.1 & 1.0 & $\cdots$ & $\cdots$ & $\cdots$ & KPNO \\
\hline 53082.674 & 0.830 & 45.7 & -0.3 & 1.0 & $\ldots$ & $\ldots$ & $\ldots$ & KPNO \\
\hline 53031.901 & 0.282 & -3.6 & -0.1 & 1.0 & $\ldots$ & $\ldots$ & $\ldots$ & Fair \\
\hline 53032.904 & 0.313 & -4.4 & 0.0 & 1.0 & $\ldots$ & $\ldots$ & $\cdots$ & Fair \\
\hline 53057.781 & 0.071 & 23.8 & 0.2 & 1.0 & $\ldots$ & $\ldots$ & $\ldots$ & Fair \\
\hline 53302.031 & 0.516 & 8.4 & 0.1 & 1.0 & $\ldots$ & $\ldots$ & $\ldots$ & Fair \\
\hline 53313.943 & 0.879 & 46.6 & 0.0 & 1.0 & $\ldots$ & $\ldots$ & $\ldots$ & Fair \\
\hline
\end{tabular}

${ }^{\mathrm{a}} \mathrm{McD}$-McDonald Observatory, KPNO—Kitt Peak National Observatory, Fair-Fairborn Observatory.

(This table is available in its entirety in machine-readable and Virtual Observatory (VO) forms.)

velocities and 0.02 to the secondary velocities. A simultaneous orbital solution of the weighted primary and secondary velocities results in the orbital elements and related parameters listed in Table 6 . The velocities are compared with the computed velocity curves in Figure 5.

In Figure 6 we plot the velocity residuals to the computed curves, determined in the combined solution, in two panels, one for the primary and one for the secondary. The secondary residuals are systematically positive, which is a reflection of the $0.8 \mathrm{~km} \mathrm{~s}^{-1}$ difference in the center-of-mass velocities of the primary and secondary. Comparison of the combined solution results with those of the separate solutions of the primary and secondary shows that the very low weights of the secondary velocities in the combined solution result in only very slight changes to the elements of the primary, well within their $1 \sigma$ uncertainties. Comparing the combined solution with that determined from the secondary velocities alone, the difference in the semiamplitude of the secondary, which directly affects the minimum masses, is at the level of $1 \sigma$, and so not significant.

Table 6 also lists the single-lined binary orbital elements from Katoh et al. (2013), which were determined from very high precision radial velocity measurements. However, we note that Griffin (2013) has concluded that the uncertainties that Katoh et al. (2013) gave in their Table 3 should be increased by a factor of 1.5 to convert their probable errors to standard errors. We find that the orbital elements of the two solutions (Table 6) are in excellent agreement. The periods differ by just $1 \sigma$, and the uncertainties for the period are essentially identical, while our uncertainties for the other elements, although very small, are about 2.0-2.5 times larger than those of Katoh et al. (2013). The addition of the solution for the 


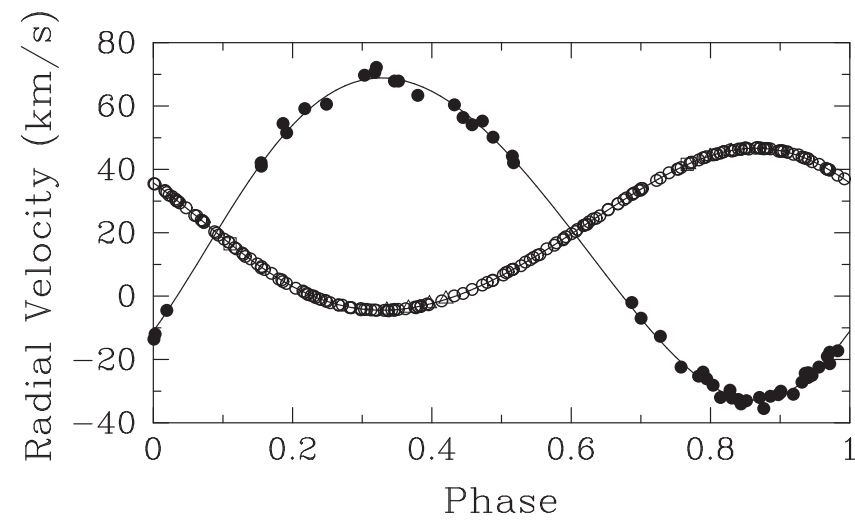

Figure 2. Radial velocities of HD 54371 compared with the computed velocity curves. Open and filled symbols represent the primary and secondary, respectively. Circles-Fairborn Observatory, triangles-KPNO, squaresMcDonald Observatory. Zero phase is a time of periastron passage.

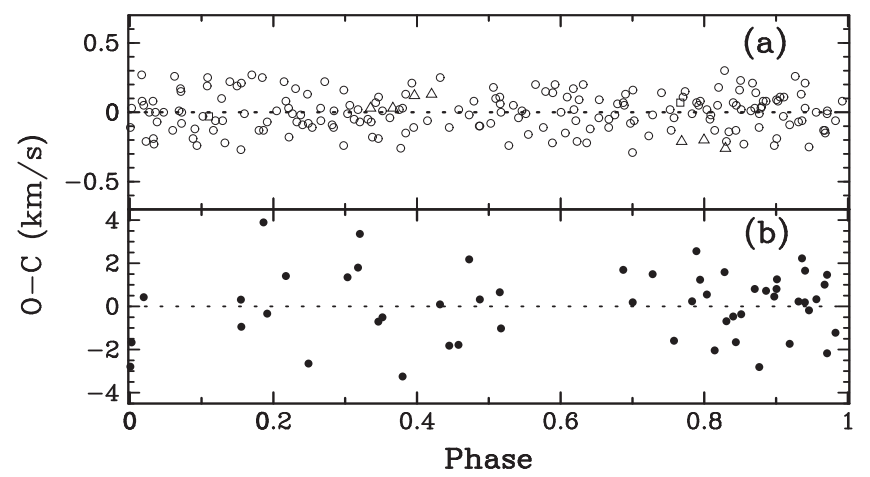

Figure 3. HD 54371 radial velocity residuals vs. orbital phase. Panel (a) shows residuals of the primary, while panel (b) is of the secondary. Circles-Fairborn Observatory, triangles - KPNO, squares-McDonald Observatory. Zero phase is a time of periastron passage.

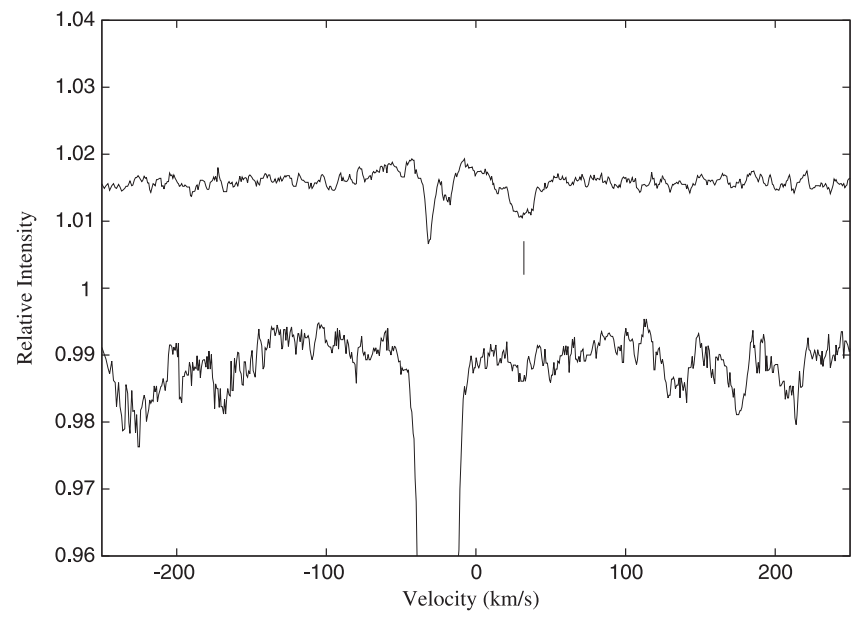

Figure 4. From a Fairborn Observatory spectrum of HR 2692, the lower solid line is the average profile of the components summed over 168 spectral regions. The upper line, arbitrarily vertically shifted for visibility, is the remainder after the average region around the primary component from all useful Fairborn spectra has been removed from the lower line. The position of the secondary is indicated with a tick mark below the summed residual spectrum.

secondary component, which enables us to directly determine a mass ratio, adds to the value of our result.

\section{3. $16 U M a$}

From 2004 through 2014 we obtained 119 Fairborn Observatory spectra (Table 7). A single McDonald Observatory spectrum from 2003 and an additional 14 observations, acquired from KPNO by Abt \& Willmarth (2006) between 2000 and 2002, extend the time baseline (Table 7). Once again, in many of our recent Fairborn spectra very weak features of the secondary have been detected after subtraction of an averaged primary feature (Figure 7).

For the primary we began by computing separate orbits for

Table 5

Radial Velocities of HR 2692

\begin{tabular}{|c|c|c|c|c|c|c|c|c|}
\hline 51957.721 & 0.648 & 8.3 & 0.0 & 1.0 & $\ldots$ & $\ldots$ & $\ldots$ & KPNO \\
\hline 51959.675 & 0.665 & 9.0 & -0.3 & 1.0 & $\ldots$ & $\ldots$ & $\ldots$ & KPNO \\
\hline 51981.671 & 0.859 & 16.7 & 0.1 & 1.0 & $\ldots$ & $\ldots$ & $\ldots$ & KPNO \\
\hline 52240.939 & 0.149 & -24.4 & 0.1 & 1.0 & $\ldots$ & $\ldots$ & $\ldots$ & KPNO \\
\hline 52241.916 & 0.158 & -24.0 & 0.1 & 1.0 & $\ldots$ & $\ldots$ & $\ldots$ & KPNO \\
\hline 52242.919 & 0.167 & -23.3 & 0.3 & 1.0 & $\ldots$ & $\ldots$ & $\ldots$ & KPNO \\
\hline 52349.642 & 0.109 & -25.5 & 0.2 & 1.0 & $\ldots$ & $\ldots$ & $\ldots$ & KPNO \\
\hline 52350.627 & 0.118 & -25.4 & 0.2 & 1.0 & $\ldots$ & $\ldots$ & $\ldots$ & KPNO \\
\hline 52370.622 & 0.294 & -14.5 & 0.0 & 1.0 & $\ldots$ & $\ldots$ & $\ldots$ & KPNO \\
\hline
\end{tabular}

${ }^{a}$ KPNO—Kitt Peak National Observatory, McD—McDonald Observatory, Fair—Fairborn Observatory.

(This table is available in its entirety in machine-readable and Virtual Observatory (VO) forms.) 
Table 6

Orbital Elements and Related Parameters of HR 2692

\begin{tabular}{|c|c|c|}
\hline Parameter & Katoh et al. $(2013)^{a}$ & This Study \\
\hline$P$ (days) & $113.23143 \pm 0.00072$ & $113.23056 \pm 0.00076$ \\
\hline$T(\mathrm{HJD})$ & $2453356.310 \pm 0.009$ & $2454262.200 \pm 0.024$ \\
\hline$e$ & $0.3870 \pm 0.0002$ & $0.38803 \pm 0.00054$ \\
\hline$\omega_{1}(\operatorname{deg})$ & $100.53 \pm 0.05$ & $100.638 \pm 0.096$ \\
\hline$K_{1}\left(\mathrm{~km} \mathrm{~s}^{-1}\right)$ & $21.242 \pm 0.006$ & $21.215 \pm 0.014$ \\
\hline$K_{2}\left(\mathrm{~km} \mathrm{~s}^{-1}\right)$ & $\ldots$ & $33.11 \pm 0.12$ \\
\hline$\gamma\left(\mathrm{km} \mathrm{s}^{-1}\right)$ & $\ldots$ & $-2.9797 \pm 0.0084$ \\
\hline$m_{1} \sin ^{3} i\left(M_{\odot}\right)$ & $\cdots$ & $0.8976 \pm 0.0068$ \\
\hline$m_{2} \sin ^{3} i\left(M_{\odot}\right)$ & $\cdots$ & $0.5751 \pm 0.0025$ \\
\hline$a_{1} \sin i\left(10^{6} \mathrm{~km}\right)$ & $30.498 \pm 0.010$ & $30.445 \pm 0.021$ \\
\hline$a_{2} \sin i\left(10^{6} \mathrm{~km}\right)$ & $\ldots$ & $47.52 \pm 0.21$ \\
\hline $\mathrm{RV} \mathrm{rms}_{1}\left(\mathrm{~km} \mathrm{~s}^{-1}\right)$ & $\ldots$ & 0.13 \\
\hline $\mathrm{RV} \mathrm{rms} 2\left(\mathrm{~km} \mathrm{~s}^{-1}\right)$ & $\cdots$ & 1.01 \\
\hline
\end{tabular}

${ }^{\mathrm{a}}$ The uncertainties of Katoh et al. (2013) have been increased by a factor of 1.5 because Griffin (2013) noted that the values quoted by Katoh et al. (2013) appear to be probable errors rather than standard errors.

the Fairborn and KPNO velocities (Abt \& Willmarth 2006). The center-of-mass velocities of the two orbits agree to within $0.1 \mathrm{~km} \mathrm{~s}^{-1}$. An additional comparison of the two solutions indicates that the velocities of Fairborn and KPNO should both be given unit weights, except for the velocities of Abt \& Willmarth (2006) from spectra obtained before JD 2452118. According to Abt \& Willmarth (2006) those velocities are significantly less precise, and we have assigned weights of 0.3 to those six values. An orbital solution of the secondary velocities results in weights of 0.01 relative to the Fairborn velocities of the primary. Given the difficulty in measuring the secondary lines, the center-of-mass velocities of the primary and secondary solutions are in reasonable agreement, differing by $0.8 \mathrm{~km} \mathrm{~s}^{-1}$. A simultaneous orbital solution of the weighted primary and secondary velocities results in the orbital elements and related parameters listed in Table 8 . The velocities are compared with the computed velocity curves in Figure 8.

The two-panel plot of the velocity residuals versus phase (Figure 9) is similar to that of Figure 6. Because of the $0.8 \mathrm{~km} \mathrm{~s}^{-1}$ difference between the center-of-mass velocities of the primary and secondary, the residual velocities of the secondary are systematically positive in the combined orbit. Comparison of the combined solution results with those of the separate solutions of the primary and secondary shows that the very low weights of the secondary velocities in the combined solution result in only very slight changes to the elements of the primary, well within their $1 \sigma$ uncertainties. Comparing the combined solution with that determined from the secondary velocities alone, the difference in the semiamplitude of the secondary, which directly affects the minimum masses, is at the level of $1 \sigma$, and so not significant.

As we did for HR 2692, we compare our orbital elements and related quantities in Table 8 with the results of Katoh et al. (2013), which were determined from very high-precision radial velocity measurements. In that table we have once again increased their listed uncertainties by a factor of 1.5 as indicated by the results of Griffin (2013). The orbital elements of the two solutions are in excellent agreement. Our period differs from theirs by just $2 \sigma$, and the uncertainties of the

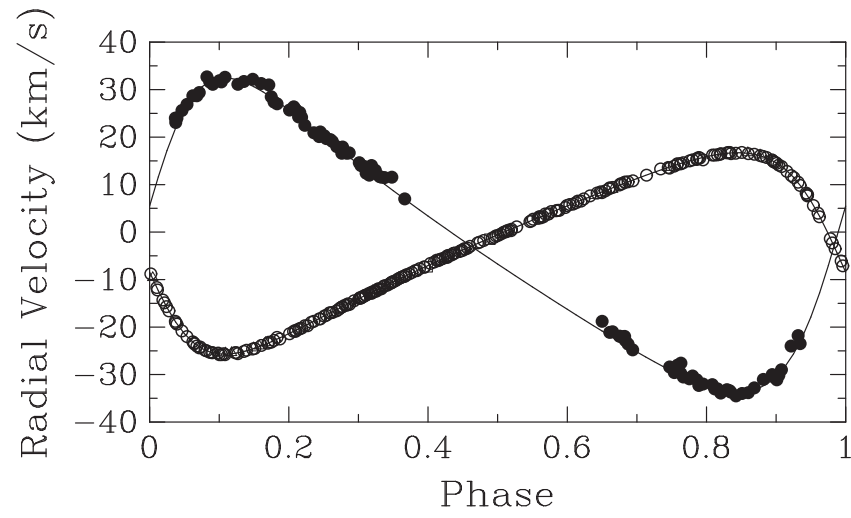

Figure 5. Radial velocities of HR 2692 compared with the computed velocity curves. Open and filled symbols represent the primary and secondary, respectively. Circles-Fairborn Observatory, triangles-KPNO, squaresMcDonald Observatory. Zero phase is a time of periastron passage.

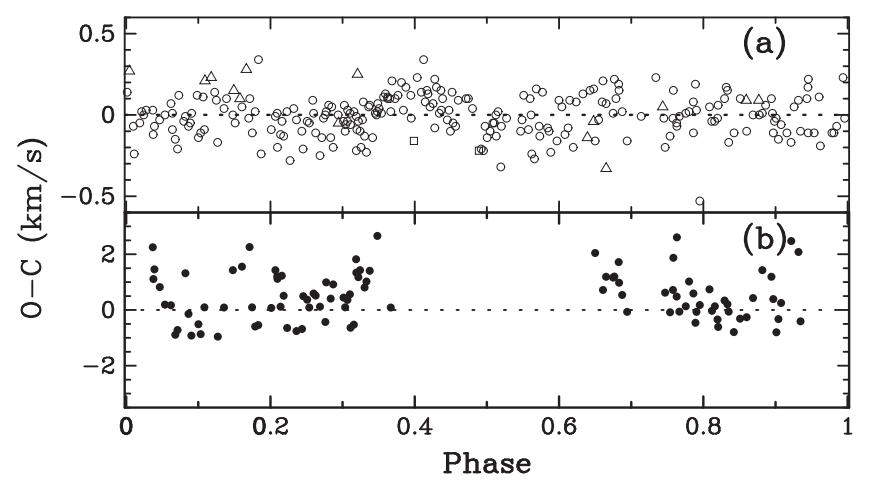

Figure 6. HR 2692 radial velocity residuals vs. orbital phase. Panel (a) shows residuals of the primary, while panel (b) is of the secondary. Circles-Fairborn Observatory, triangles - KPNO, squares-McDonald Observatory. Zero phase is a time of periastron passage.

period determination are essentially identical. Our uncertainties for the other elements, although very small, are about 2.0-2.5 times larger than those of Katoh et al. (2013). The addition of the solution for the secondary component provides the binary mass ratio and significantly enhances the value of our result.

\section{SPECTRAL TYPE AND MAGNITUDE DIFFERENCE}

From spectra obtained at KPNO Strassmeier \& Fekel (1990) identified several luminosity-sensitive and temperature-sensitive line ratios in the 6430-6465 Å region. They used those critical line ratios as well as the general appearance of the spectrum around $6430 \AA$ as spectral-type criteria. The luminosity sensitive lines in this region are useful for stars later in spectral class than early-G, but those lines lose their luminosity sensitivity for hotter stars. Our Fairborn Observatory echelle spectra include the $\mathrm{Ho}$ line. For solar-type dwarfs that line has significant wings, while for subgiants the wings are substantially reduced. So both the spectral and luminosity classes can be determined from our various spectra. The luminosity class of each star can also be determined by another method. First, its absolute visual magnitude is computed from the Hipparcos parallax. Then that magnitude, converted to a luminosity, and the adopted temperature of the star can be compared to evolutionary tracks in an $\mathrm{H}-\mathrm{R}$ diagram.

The KPNO TI CCD spectra of our binaries have been compared with spectra of a variety of F, G, and K dwarfs and 
Table 7

Radial Velocities of $16 \mathrm{UMa}$

\begin{tabular}{|c|c|c|c|c|c|c|c|c|}
\hline $\begin{array}{l}\text { Hel. Julian Date } \\
\text { (HJD_-2400000) }\end{array}$ & Phase & $\begin{array}{c}V_{1} \\
\left(\mathrm{~km} \mathrm{~s}^{-1}\right)\end{array}$ & $\begin{array}{c}(O-C)_{1} \\
\left(\mathrm{~km} \mathrm{~s}^{-1}\right)\end{array}$ & $\mathrm{Wt}_{1}$ & $\begin{array}{c}V_{2} \\
\left(\mathrm{~km} \mathrm{~s}^{-1}\right)\end{array}$ & $\begin{array}{c}(O-C)_{2} \\
\left(\mathrm{~km} \mathrm{~s}^{-1}\right)\end{array}$ & $\mathrm{Wt}_{2}$ & Source $^{\mathrm{a}}$ \\
\hline 51885.034 & 0.707 & 15.2 & -0.4 & 0.3 & $\ldots$ & $\ldots$ & $\ldots$ & KPNO \\
\hline 51887.018 & 0.829 & -2.3 & -0.1 & 0.3 & $\cdots$ & $\cdots$ & $\cdots$ & KPNO \\
\hline 51959.758 & 0.308 & -24.0 & 0.2 & 0.3 & $\cdots$ & $\cdots$ & $\cdots$ & KPNO \\
\hline 51981.744 & 0.662 & 17.8 & 0.1 & 0.3 & $\cdots$ & $\ldots$ & $\ldots$ & KPNO \\
\hline 51983.721 & 0.784 & 6.9 & 0.4 & 0.3 & $\cdots$ & $\ldots$ & $\cdots$ & KPNO \\
\hline 52349.758 & 0.324 & -20.9 & 0.3 & 1.0 & $\ldots$ & $\ldots$ & $\ldots$ & KPNO \\
\hline 52351.732 & 0.445 & 0.8 & 0.0 & 1.0 & $\ldots$ & $\ldots$ & $\ldots$ & KPNO \\
\hline 52370.709 & 0.614 & 17.4 & 0.0 & 1.0 & $\ldots$ & $\ldots$ & $\cdots$ & KPNO \\
\hline 52371.658 & 0.672 & 17.2 & -0.2 & 1.0 & $\ldots$ & $\ldots$ & $\cdots$ & KPNO \\
\hline 52372.685 & 0.736 & 13.2 & 0.1 & 1.0 & $\ldots$ & $\ldots$ & $\ldots$ & KPNO \\
\hline 52373.693 & 0.798 & 4.3 & 0.2 & 1.0 & $\cdots$ & $\cdots$ & $\cdots$ & KPNO \\
\hline
\end{tabular}

${ }^{a}$ KPNO—Kitt Peak National Observatory, McD—McDonald Observatory, Fair-Fairborn Observatory.

(This table is available in its entirety in machine-readable and Virtual Observatory (VO) forms.)

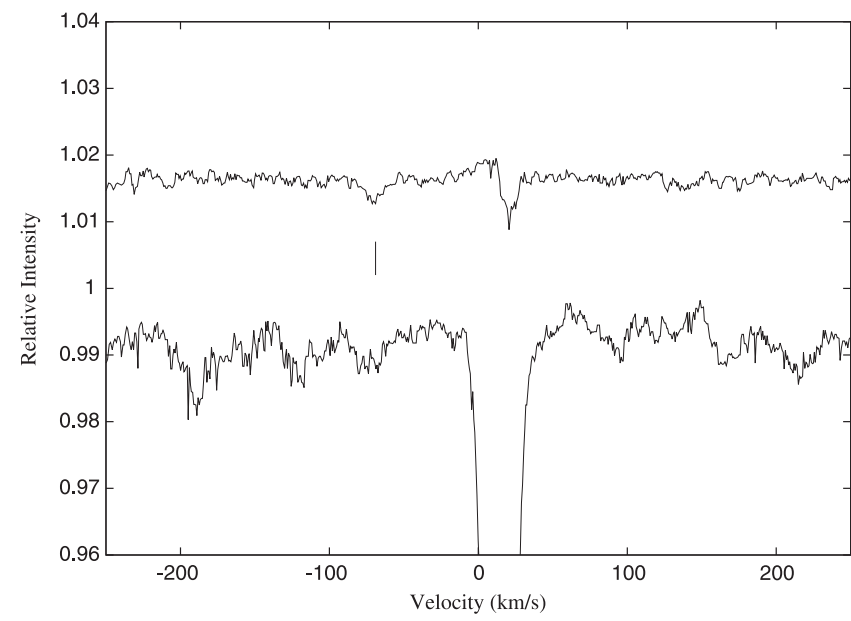

Figure 7. From a Fairborn Observatory spectrum of $16 \mathrm{UMa}$, the lower solid line is the average profile of the components summed over 168 spectral regions. The upper line, arbitrarily vertically shifted for visibility, is the remainder after the average region around the primary component from all useful Fairborn spectra has been removed from the lower line. The position of the secondary is indicated with a tick mark below the summed residual spectrum.

subgiants from the lists of Keenan \& McNeil (1989) and Fekel (1997). The spectra of such reference stars were acquired at KPNO with the same telescope, spectrograph, and detector as our binary star spectra. With a computer program developed by Huenemoerder \& Barden (1984) and Barden (1985) a variety of reference star spectra were rotationally broadened when necessary, shifted in radial velocity, and compared with the program star spectrum to determine the best spectral type match.

From the equivalent widths of the average summed features of the primary and secondary in several of our highest $\mathrm{S} / \mathrm{N}$ Fairborn spectra, we have estimated the continuum intensity ratio of the binary components at $6000 \AA$, which we convert into an estimated $V$ magnitude difference. If the components are main sequence stars of very different spectral types, as is
Table 8

Orbital Elements and Related Parameters of $16 \mathrm{UMa}$

\begin{tabular}{lcc}
\hline \hline Parameter & Katoh et al. $(2013)^{\mathrm{a}}$ & \multicolumn{1}{c}{ This Study } \\
\hline$P$ (days) & $16.23966 \pm 0.00002$ & $16.239631 \pm 0.000015$ \\
$T$ (HJD) & $243400.081 \pm 0.004$ & $2454358.214 \pm 0.013$ \\
$e$ & $0.1055 \pm 0.0002$ & $0.10635 \pm 0.00054$ \\
$\omega_{1}(\mathrm{deg})$ & $137.41 \pm 0.10$ & $137.18 \pm 0.29$ \\
$K_{1}\left(\mathrm{~km} \mathrm{~s}^{-1}\right)$ & $35.305 \pm 0.008$ & $35.344 \pm 0.018$ \\
$K_{2}\left(\mathrm{~km} \mathrm{~s}^{-1}\right)$ & $\ldots$ & $64.97 \pm 0.27$ \\
$\gamma\left(\mathrm{km} \mathrm{s}^{-1}\right)$ & $\ldots$ & $-14.731 \pm 0.014$ \\
$m_{1} \sin ^{3} i\left(M_{\odot}\right)$ & $\ldots$ & $1.0817 \pm 0.0096$ \\
$m_{2} \sin ^{3} i\left(M_{\odot}\right)$ & $\ldots$ & $0.5884 \pm 0.0030$ \\
$a_{1} \sin i\left(10^{6} \mathrm{~km}^{2}\right.$ & $7.840 \pm 0.002$ & $7.8479 \pm 0.0041$ \\
$a_{2} \sin i\left(10^{6} \mathrm{~km}\right)$ & $\ldots$ & $14.427 \pm 0.060$ \\
$\mathrm{RV} \mathrm{rms}_{1}\left(\mathrm{~km} \mathrm{~s}^{-1}\right)$ & $\ldots$ & 0.15 \\
$\mathrm{RV} \mathrm{rms}_{2}\left(\mathrm{~km} \mathrm{~s}^{-1}\right)$ & $\ldots$ & 1.66 \\
\hline
\end{tabular}

${ }^{a}$ The uncertainties of Katoh et al. (2013) have been increased by a factor of 1.5 because Griffin (2013) noted that the values quoted by Katoh et al. (2013) appear to be probable errors rather than standard errors.

the case for HD 54371 and $16 \mathrm{UMa}$, then comparing the spectra of the individual stars, the cooler secondary lines are intrinsically stronger than those of the primary. In that case, in the combined spectrum the continuum intensity ratio becomes a minimum magnitude difference. We conclude below that the primary of our third star, HR 2692 is a cool subgiant. Compared to its spectrum while on the main sequence, its spectral lines will have increased in strength as it has evolved to lower temperatures. Thus, the intrinsic line strength of the lower-mass secondary may be similar to that of the primary.

The difficulty in measuring the extremely weak secondary lines results in a large estimated uncertainty for the magnitude difference of each binary. But because the magnitude 


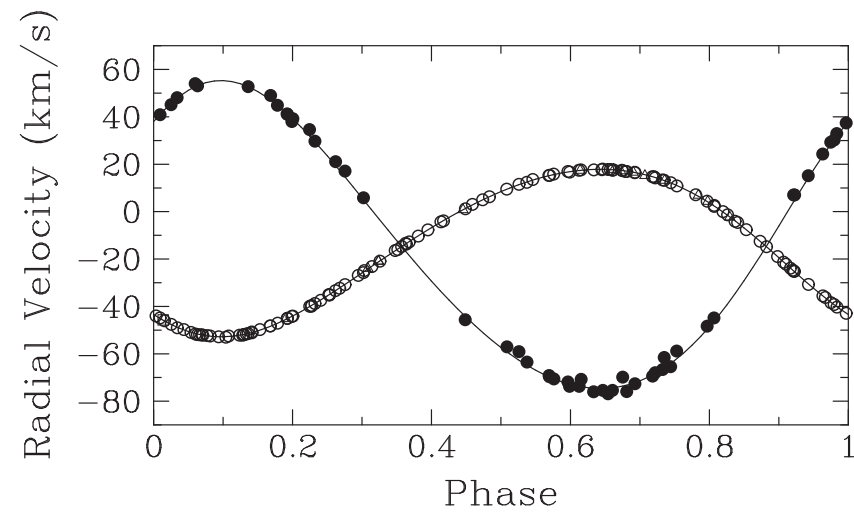

Figure 8. Radial velocities of 16 UMa compared with the computed velocity curves. Open and filled symbols represent the primary and secondary, respectively. Circles-Fairborn Observatory, triangles-KPNO, squaresMcDonald Observatory. Zero phase is a time of periastron passage.

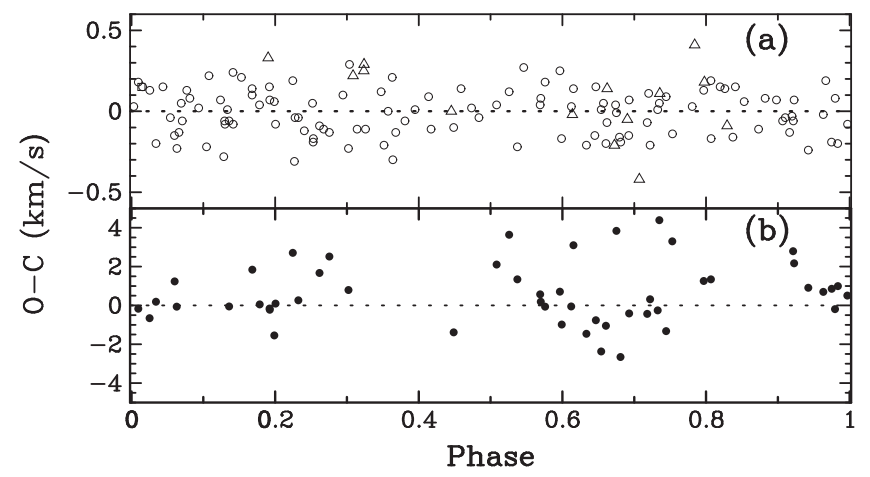

Figure 9. 16 UMa radial velocity residuals vs. orbital phase. Panel (a) shows residuals of the primary, while panel (b) is of the secondary. Circles-Fairborn Observatory, triangles-KPNO, squares-McDonald Observatory. Zero phase is a time of periastron passage.

difference is very large, when the total magnitude of the binary is converted into the individual magnitudes of the components, that uncertainty will have a minimal effect on the magnitude of the primary but make the magnitude of the secondary very uncertain.

\subsection{HD 54371}

The spectrum of HD 54371 is well matched by that of $61 \mathrm{Vir}$ (G6 V, Gray et al. 2003, $[\mathrm{Fe} / \mathrm{H}]=0.01$ Taylor 2005). Our spectral type of $\mathrm{G} 6 \mathrm{~V}$ is in good agreement with earlier classifications that range from $\mathrm{G} 5 \mathrm{~V}$ (Abt 1985) to G8 V (Harlan 1969). The comparison with $61 \mathrm{Vir}$ also indicates that the iron abundance of HD 54371 is close to the solar value, in agreement with the result of Gray et al. (2003).

The secondary to primary equivalent width ratio of the averaged summed lines of the two components in several of our Fairborn spectra is 0.02 , which results in a minimum magnitude difference $\Delta V=4.2$. We estimate that the actual magnitude difference is $4.6 \pm 0.6$.

\section{2. $H R 2692$}

The spectrum of HR 2692 was compared to both dwarfs and subgiants. For the dwarfs the broader wings of their strong lines in the $6430 \AA$ region clearly do not match the corresponding features in HR 2692. On the other hand the subgiant $\beta$ Aql (G8 IV, Keenan \& McNeil 1989, $[\mathrm{Fe} / \mathrm{H}]=-0.10 \mathrm{Kang}$ et al. 2011) is a very good fit although some of its lines are a bit too weak suggesting that the Fe abundance of HR 2692 is closer to the solar value. The $\mathrm{H} \alpha$ line in our Fairborn spectra shows little or no wings and thus indicates that HR 2962 is not a dwarf. The Hipparcos parallax positions the star at the red side of the Hertzsprung gap in the subgiant region of the $\mathrm{H}-\mathrm{R}$ diagram. Thus, our G8 IV classification confirms the proposed subgiant luminosity class of Beavers \& Salzer (1985).

The secondary to primary ratio of the equivalent widths from a few of our best Fairborn spectra is 0.035 . This results in a magnitude difference of $\Delta V=3.6 \pm 0.6$, which we adopt as our best estimate based on the mass and expected magnitude of the secondary.

\section{3. $16 U M a$}

An excellent fit to the spectrum of $16 \mathrm{UMa}$ is provided by $\iota$ Per (G0 V, Johnson \& Morgan 1953, $[\mathrm{Fe} / \mathrm{H}]=0.07$ Taylor 2005), while the fit with $\lambda$ Ser (G0 V, Keenan \& McNeil 1989, $[\mathrm{Fe} / \mathrm{H}]=0.0$ Taylor 2005) produces lines that are slightly too weak. An examination of the $\mathrm{H} \alpha$ line of $16 \mathrm{UMa}$ in our Fairborn spectra, shows that that feature has the broad wings expected for a dwarf. This result is confirmed by its Hipparcos parallax, which also indicates that the star is a dwarf. Our spectral type of G0 V is in accord with earlier classifications that, as noted in Section 2, included F9 V, G0 V, and G0 IV-V. Our best fit spectrum has an iron abundance that is slightly elevated in value compared to the Sun, reasonably consistent with abundance analyses such as those of Chen et al. (2000) and Takeda et al. (2005), which indicate that $16 \mathrm{UMa}$ has a slightly sub-solar or solar iron abundance.

From several of our Fairborn spectra, we estimate the secondary to primary equivalent width ratio to be 0.025 . This results in a minimum magnitude difference $\Delta V=4.0$. We estimate that the true magnitude difference to be $4.5 \pm 0.6$.

\section{ASTROMETRIC ORBITS}

In the Hipparcos and Tycho Catalogues (Perryman \& ESA 1997), out of the three systems considered here, only HR 2692 was processed with an orbital model, while the others were treated as single stars. Their astrometric model of HR 2692 partly adopted the spectroscopic orbit of Beavers \& Salzer (1985). Using better assessment criteria (Pourbaix \& Arenou 2001; Pourbaix \& Boffin 2003), Jancart et al. (2005) reexamined the three systems. To avoid too many false positives, they adopted an extremely stringent threshold $(0.006 \%)$ for their probability tests. Jancart et al. (2005) confirmed the astrometric solution for HR 2692 and retained the single star solutions for the other two binaries. With the very same criteria and our revised spectroscopic solutions, we would conclude that Hipparcos did not notice any wobble for our three systems.

\subsection{HD 54371}

We examined the Hipparcos data for HD 54371 and found no evidence of any astrometric displacement of its photocenter. Thus, we are unable to determine an orbital inclination from the astrometric data. 


\subsection{HR 2692}

With our new spectroscopic solution of the orbital elements of HR 2692, we imposed our spectroscopic values of the period, $P$, the eccentricity, $e$, longitude of periastron, $\omega$, and time of periastron, $T$ (Table 6) on the Hipparcos data to compute a new astrometric solution. Returning to the original criteria of Pourbaix \& Boffin (2003), we confirm the astrometric solution for HR 2692. In our new solution, our values for $a_{0}$, the semimajor axis of the photocentric orbit, $i$, the orbital inclination, and $\Omega$, the position angle of the line of nodes, are $4.9 \pm 0.3 \mathrm{mas}, 66.0^{\circ} \pm 6.2^{\circ}$, and $77.0^{\circ} \pm 6.6^{\circ}$, respectively. Our values for $a_{0}$ and $i$ are very similar to those of the Jancart et al. (2005) solution, while $\Omega$ has decreased by nearly $9^{\circ}$, resulting in just a $1.3 \sigma$ difference.

\section{3. $16 U M a$}

The binary 16 UMa was one of 282 stars that Jancart et al. (2005) initially flagged as an astrometric binary. However, it did not pass their more stringent tests, and so they did not attempt to obtain an astrometric solution from the Hipparcos data. However, returning to the criteria of Pourbaix \& Boffin (2003), we solve for its astrometric orbit. Once again, we adopted our new spectroscopic values for $P, e, \omega$, and $T$ and determined the results for $a_{0}, i$, and $\Omega$ as well as the parallax and proper motions (Table 9). With a value of $i$ of $106^{\circ} \pm 12^{\circ}$, the orbital inclination is high. Owing to the short orbital period of $16 \mathrm{UMa}$, neither the parallax nor the proper motion is substantially revised with respect to the published values.

\section{BASIC PROPERTIES}

\subsection{HD 54371}

We use the Stefan-Boltzmann law to determine the basic properties of HD 54371. We start by adopting a $V$ magnitude of 7.09 and a $B-V$ color of $0.700 \mathrm{mag}$ from the Hipparcos catalog (Perryman \& ESA 1997). With our adopted $V$ magnitude difference of $4.6 \pm 0.6$, the individual $V$ magnitude of the primary is $7.11 \pm 0.05$. The parallax from the new Hipparcos parallax reduction by van Leeuwen (2007) is 39.73 \pm 0.54 mas and corresponds to a distance of $25.2 \pm 0.4 \mathrm{pc}$. Being such a nearby system, we assume that the interstellar extinction is negligible. The resulting absolute magnitude of the primary is $M_{V}=5.11 \pm 0.06 \mathrm{mag}$. Assuming the $B-V$ color of the combined system for the primary, we obtain its effective temperature and bolometric correction from Table 3 of Flower (1996). From spectral type and temperature calibration uncertainties, we estimate an effective temperature uncertainty of $\pm 100 \mathrm{~K}$. Those results produce a luminosity of the primary, $L_{1}$, of $0.79 \pm 0.04 L_{\odot}$ and a radius of the primary, $R_{1}$, of $0.96 \pm 0.04 R_{\odot}$.

Estimates of the secondary's parameters are more problematic because its spectrum is barely detectable in our observations. From our estimated magnitude difference we find a $V$ mag of 11.71 and from its minimum mass we adopt a $B-V$ of 1.48. With the revised Hipparcos parallax, its absolute magnitude becomes $9.7 \pm 0.6$. We then adopt its effective temperature and bolometric correction from Flower (1996) and estimate a temperature uncertainty of $\pm 200 \mathrm{~K}$. As a result, we obtain a luminosity of the secondary, $L_{2}$, of 0.03 $\pm 0.02 L_{\odot}$ and a radius, $R_{2}$, of $0.35 \pm 0.10 R_{\odot}$.
With our estimated effective temperature and luminosity for the primary of HD 54371 its position in the H-R diagram is compared with the solar-abundance evolutionary tracks of Girardi et al. (2000) in Figure 10. This comparison suggests a mass of $0.9 M_{\odot}$ with an uncertainty that includes $1.0 M_{\odot}$. From our orbital solution the minimum mass of the primary is $1.0 M_{\odot}$ (Table 4), the same as the upper limit of its mass from the evolutionary track comparison, which suggests that the orbital inclination is high and approaches $90^{\circ}$. With a mass of $0.5 M_{\odot}$ (Table 4) the secondary is an early $\mathrm{M}$ dwarf (Table B1, Gray 1992).

\subsection{HR 2692}

We determine the properties of HR 2692 with the use of the Stefan-Boltzmann law. First, from the Hipparcos catalog (Perryman \& ESA 1997) we adopt a $V$ magnitude of 6.43 and $B-V$ of $0.880 \mathrm{mag}$ for the combined system. Our estimated $V$ magnitude difference of $3.6 \pm 0.6$ produces a $V$ magnitude of $6.47 \pm 0.05$ for the primary. With the parallax of $21.82 \pm 0.40$ mas (van Leeuwen 2007) and no correction for interstellar reddening, its absolute magnitude $M_{V}$ is $3.16 \pm 0.06$. Adopting the combined $B-V$ for that of the primary, we obtain its effective temperature and bolometric correction from Table 3 of Flower (1996). From spectral type and temperature calibration uncertainties, we estimate an effective temperature uncertainty of $\pm 100 \mathrm{~K}$. Those results are used to determine the luminosity and radius of the primary, $L_{1}=5.4 \pm 0.3 L_{\odot}$ and, $R_{1}$ $=3.0 \pm 0.1 R_{\odot}$.

In a similar manner for the much more uncertain secondary star parameters we begin by determining $V=10.1 \pm 0.6 \mathrm{mag}$ and from the system's parallax an absolute magnitude $M_{V}$ $=6.8 \pm 0.6$ mag. Its estimated mass corresponds to a $\mathrm{K} 2 \mathrm{~V}$ star, and so we assume $B-V=0.912$ (Table B1, Gray 1992). We then adopt its effective temperature and bolometric correction from Flower (1996) and estimate a temperature uncertainty of $\pm 300 \mathrm{~K}$. The resulting luminosity and radius of the secondary are $L_{2}=0.2 \pm 0.1 L_{\odot}$ and $R_{2}=0.6 \pm 0.2 R_{\odot}$.

The primary is plotted in an $\mathrm{H}-\mathrm{R}$ diagram (Figure 10), and its position is compared with the solar-abundance evolutionary tracks of Girardi et al. (2000), which suggest a mass of $1.4 M_{\odot}$. From our minimum masses of HR 2692 and the astrometric inclination of $66^{\circ} \pm 6^{\circ}$ we obtain masses of 1.18 and $0.75 M_{\odot}$ for the components. When the inclination uncertainty is considered, the mass of the primary ranges from 1.04 to 1.38 $M_{\odot}$, nearly reaching the mass suggested by the solar-abundance evolutionary tracks. The mass of the secondary ranges from 0.67 to $0.88 M_{\odot}$ and so indicates that it is a late-G or $\mathrm{K}$ dwarf (Table B1, Gray 1992).

\section{3. $16 U M a$}

As we did for the other two binaries, we determine the basic properties of 16 UMa by using the Stefan-Boltzmann law. For the combined system we first adopt a $V$ magnitude of 5.18 and a $B-V$ color of 0.605 mag from the Hipparcos catalog (Perryman \& ESA 1997). Our estimated $V$ magnitude difference of $4.5 \pm 0.6$ results in $V=5.20 \pm 0.05$ for the primary. The system is even closer to the Sun than HD 54371 and has a parallax of $51.20 \pm 0.74$ mas (Table 9), which corresponds to a distance of $19.5 \pm 0.3 \mathrm{pc}$. The primary's absolute magnitude is $M_{V}=3.75 \pm 0.06$. We assume that the primary's $B-V$ color is the same as that of the combined 
Table 9

Astrometric Orbital Solution of $16 \mathrm{UMa}^{\mathrm{a}}$

\begin{tabular}{lcc}
\hline \hline Parameter & Symbol & Value \\
\hline Parallax & $\varpi(\mathrm{mas})$ & $51.2 \pm 0.74$ \\
Proper motion in Right Ascension & $\mu_{\alpha^{*}}\left(\mathrm{mas} \mathrm{yr}^{-1}\right)$ & $-9.1 \pm 0.4$ \\
Proper motion in declination & $\mu_{\delta}\left(\mathrm{mas} \mathrm{yr}^{-1}\right)$ & $-31.6 \pm 0.5$ \\
Photocentric semimajor axis & $a_{0}(\mathrm{mas})$ & $2.9 \pm 0.2$ \\
Inclination & $i(\mathrm{deg})$ & $106.0 \pm 12.0$ \\
Position angle of the line of nodes & $\Omega(\mathrm{deg})$ & $107.0 \pm 14.0$
\end{tabular}

a The values of $P, T, e$, and $\omega$ have been adopted from the spectroscopic solution in Table 8.

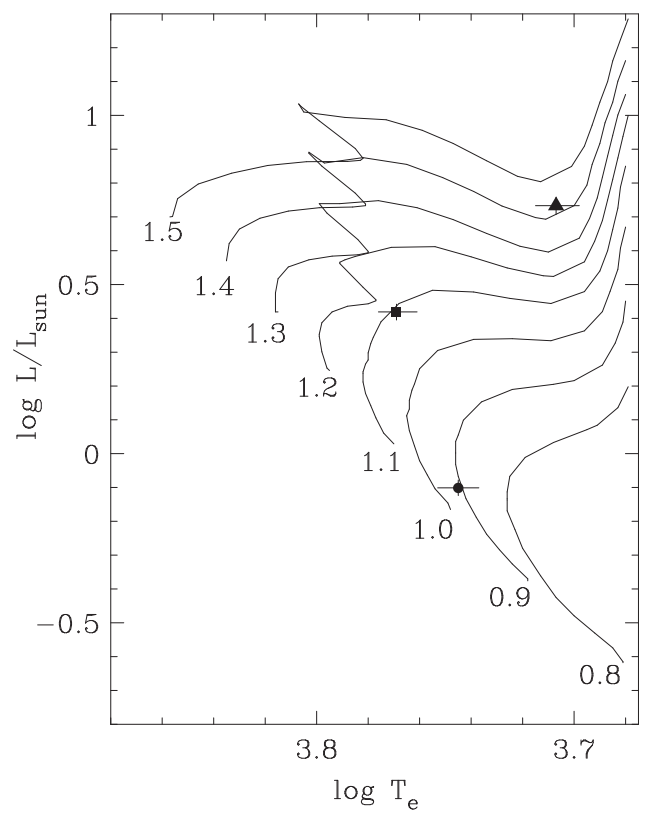

Figure 10. Positions of the primary components of HD 54371 (circle), HR 2692 (triangle), and $16 \mathrm{UMa}$ (square) compared with the $0.8-1.5 M_{\odot}$ solarabundance evolutionary tracks of Girardi et al. (2000). Our estimated uncertainties are shown.

system and use Table 3 of Flower (1996) to provide the bolometric correction and effective temperature that we adopt for the star. The effective temperature uncertainty is estimated to be $\pm 100 \mathrm{~K}$. Then for the primary we obtain a luminosity of $L_{1}=2.6 \pm 0.1 L_{\odot}$ and a radius of $R_{1}=1.6 \pm 0.1 R_{\odot}$.

We similarly determine the parameters for the secondary, which are very approximate because of the large magnitude difference of $4.5 \pm 0.6$. The $V$ magnitude of the secondary becomes $9.7 \pm 0.6$ and from the parallax of the sytem its absolute magnitude $M_{V}$ is then $8.2 \pm 0.6$. Using Table B1 of Gray (1992), from the absolute magnitude of the primary and the magnitude difference we adopt a $B-V$ of 1.31 for the secondary. The effective temperature and the bolometric correction again come from Flower (1996), and we adopt a temperature uncertainty of $\pm 200 \mathrm{~K}$. The resulting luminosity and radius for the secondary are $L_{2}=0.08 \pm 0.04 L_{\odot}$ and $R_{2}=0.50 \pm 0.14 R_{\odot}$.

Our estimated temperature and luminosity for the primary are plotted in an H-R diagram (Figure 10) and compared to the solar-abundance evolutionary tracks of Girardi et al. (2000). The position of the primary in the H-R diagram confirms that it is a dwarf and suggests a mass of $1.1 M_{\odot}$. Combining its minimum mass of $1.08 M_{\odot}$ (Table 8 ) with the inclination from our astrometric orbit, $106^{\circ} \pm 12^{\circ}$, results in a mass of $1.22 M_{\odot}$. But the large uncertainty produces a range from 1.09 to 1.57 $M_{\odot}$. Thus, the mass from the evolutionary tracks is best reconciled with a mass resulting from an orbital inclination near $90^{\circ}$. For the secondary the orbital inclination increases its minimum mass from $0.59 M_{\odot}$ to $0.66 M_{\odot}$ and suggests that the secondary is a mid- to late-K dwarf (Table B1, Gray 1992).

\section{CIRCULARIZATION AND SYNCHRONIZATION}

The theories of Zahn (1977) and Tassoul \& Tassoul (1992) rely on very different mechanisms to explain the way that binary orbits become circularized and binary components attain rotational synchronization. In both theories the timescales for circularization and synchronization are proportional to the fractional separation $a / R$ raised to some large power. In this ratio $a$ is the orbit semimajor axis and $R$ is the radius of the star. For synchronization the exponent of the ratio ranges from 4 to 6 depending on the theory (Tassoul \& Tassoul 1996). Of course, the semimajor axis is related to the orbital period by Kepler's third law. Despite the rather different absolute times scales predicted by the two theories, both theories produce the conclusion that rotational synchronization occurs before orbital circularization.

Our orbits are still eccentric. In such an orbit Hut (1981) has shown that the rotational angular velocity of a star will tend to synchronize with that of the orbital motion at periastron, a condition called pseudosynchronous rotation. With the use of Equation (42) of Hut (1981) we compute pseudosynchronous rotation periods of 31.9 days for HD 54371, 57.5 days for HR 2692, and 15.2 days for 16 UMa.

To examine whether the stars in our three systems are pseudosynchronously rotating, we have determined their projected rotational velocities with the procedure of Fekel (1997) from our KPNO red-wavelength spectra with the TI CCD detector. For the late-F and $\mathrm{G}$ stars, following Fekel (1997), a macroturbulent broadening of $3 \mathrm{~km} \mathrm{~s}^{-1}$ has been adopted. From our spectra, the projected rotational velocities of the primaries for HD 54371, HR 2692, and 16 UMa are $5.1 \pm$ $1.0,1.2 \pm 2.0$, and $6.0 \pm 1.0 \mathrm{~km} \mathrm{~s}^{-1}$, respectively. The estimated uncertainty for HR 2692 is doubled because the rotational broadening is not the dominant broadening source and is significantly less than the adopted macroturbulence. Because of the extreme weakness of the secondary lines, we have not attempted to measure their projected rotational velocities as such velocities would have very large uncertainties.

To obtain equatorial rotational velocities from the $v \sin i$ values we assume, as is generally done, that the axes of the orbital and rotational planes are parallel. If the two inclinations are equal, then we can adopt the orbital inclination as the rotational inclination.

\subsection{HD 54371}

To determine whether the primary of HD 54371 is rotating pseudosynchronously, we first compute its equatorial velocity from our projected rotational velocity and then compare it with the predicted pseudosynchronous velocity. Its $v \sin i$ value is $5.1 \pm 1.0 \mathrm{~km} \mathrm{~s}^{-1}$. A comparison of the primary's minimum mass with its mass from evolutionary tracks suggests that the orbital inclination is close to $90^{\circ}$. Therefore, we simply adopt 
the projected rotational velocity as the equatorial velocity. From the Stefan-Boltzmann law a radius of $0.94 R_{\odot}$ and pseudosynchronous rotation period of 31.9 days we compute a pseudosynchronous rotational velocity of $1.5 \mathrm{~km} \mathrm{~s}^{-1}$. Thus, the primary appears to be rotating faster than its pseudosynchronous velocity. It may not have achieved the slower pseudosynchronous rotation because, as suggested by its position in Figure 10, it is a relatively young system.

\subsection{HR 2692}

For the primary of HR 2692 our $v \sin i$ value is $1.2 \pm$ $2.0 \mathrm{~km} \mathrm{~s}^{-1}$. From our astrometric orbit we adopt an inclination of $66^{\circ} \pm 6^{\circ}$. Thus, the equatorial velocity is $1.3 \mathrm{~km} \mathrm{~s}^{-1}$. Adopting a radius of $3.0 R_{\odot}$ from the Stefan-Boltzmann law and the pseudosynchronous rotation period of 57.5 days results in a pseudosynchronous velocity of $2.6 \mathrm{~km} \mathrm{~s}^{-1}$. Given the relatively large uncertainties, although the pseudosynchronous velocity is twice the observed one, it is certainly possible that the primary is pseudosynchronously rotating. Its position on the red side of the Hertzsprung gap and near the base of the first ascent red giant branch means that the star has significantly increased its radius and so pseudosynchronous rotation may be a reasonable expectation.

\section{3. $16 U M a$}

Our $v \sin i$ value for the primary of $16 \mathrm{UMa}$ is $6.0 \pm$ $1.0 \mathrm{~km} \mathrm{~s}^{-1}$. We adopt the inclination of $106^{\circ}$ from the astrometric orbit and so obtain $6.2 \mathrm{~km} \mathrm{~s}^{-1}$ for the equatorial velocity, while the uncertainty of just the inclination, $\pm 12^{\circ}$, produces a range from 6.0 to $6.8 \mathrm{~km} \mathrm{~s}^{-1}$. With an adopted radius of $1.6 R_{\odot}$ and pseudosynchronous period of 15.2 days, the pseudosynchronous rotational velocity is $5.3 \mathrm{~km} \mathrm{~s}^{-1}$. Thus, the observed rotational velocity of the primary is consistent with pseudosynchronous rotation. Of our three binaries, $16 \mathrm{UMa}$ has the shortest pseudosynchronous period and although on the main sequence, it appears to be somewhat evolved from the zero-age main sequence (Figure 10). Thus, pseudosynchronous rotation is a reasonable expectation.

\section{CONCLUSIONS}

Subtracting an average of the primary lines from our Fairborn Observatory spectra has enabled us to detect very weak features of the secondary in the residual spectra for three previously known single-lined spectroscopic binaries, HD 54371, HR 2692, and $16 \mathrm{UMa}$, and to determine for the first time orbital elements for both components of those systems. At visual wavelengths this spectrum subtraction has enabled us to measure secondary to primary mass ratios as small as 0.5 . Despite the significant uncertainties of the secondary velocities, we have determined minimum masses, $m \sin ^{3} i$ values, that have accuracies of $\leqslant 1.0 \%$. Astrometric orbits from the Hipparcos data and our new spectroscopic elements have been computed for HR 2692 and 16 UMa resulting in orbital inclinations for those systems. We confirm the conclusion of Beavers \& Salzer (1985) that the primary of HR 2692 is a subgiant. The primaries of HR 2692 and 16 UMa may be rotating pseudosynchronously, but that of HD 54371 is rotating faster than its pseudosynchronous velocity.

Our systems have eccentric rather than circular orbits. Thus, following McAlister (1976), to estimate the resolvability of our systems, we have determined the greater nodal separation for each binary (e.g., McAlister 1976; Halbwachs 1981). We find that those separations for HD 54371, HR 2692, and 16 UMa are 9 , 12, and 8 mas, respectively, and therefore, are within the resolution range of modern interferometers. The large magnitude differences between the primaries and secondaries can be reduced for at least two of the systems by observing in the infrared. Thus, interferometric observations with the CHARA array on Mount Wilson would complement our spectroscopic work and result in accurate three-dimensional orbits, masses, and distances for the systems allowing improved comparisons with evolutionary tracks.

We thank Helmut Abt for sharing his unpublished velocities. We also thank the referee for suggestions that improved the paper. NSF grant 1039522 from the Major Research Instrumentation Program, awarded to Tennessee State University, made much of this research possible. In addition, astronomy at Tennessee State University is supported by the state of Tennessee through its Centers of Excellence program.

\section{REFERENCES}

Abt, H. A. 1970, ApJS, 19, 387

Abt, H. A. 1985, ApJS, 59, 95

Abt, H. A. 2009, ApJS, 180, 117

Abt, H. A., \& Levy, S. G. 1976, ApJS, 30, 273

Abt, H. A., \& Willmarth, D. 2006, ApJS, 162, 207

Barden, S. C. 1985, ApJ, 295, 162

Barker, E. S., Evans, D. S., \& Laing, J. D. 1967, RGOB, 130, 355

Batten, A. H., Fletcher, J. M., \& MacCarthy, D. G. 1989, PDAO, 17, 1

Beavers, W. I., \& Salzer, J. J. 1985, PASP, 97, 355

Boden, A. F., Torres, G., \& Latham, D. W. 2006, ApJ, 644, 1193

Chen, Y. Q., Nissen, P. E., Zhao, G., Zheng, H. W., \& Benoni, T. 2000, A\&AS, 141, 491

Cowley, A. P. 1976, PASP, 88, 522

Cowley, A. P., \& Bidelman, W. P. 1979, PASP, 91, 83

Cowley, A. P., Hiltner, W. A., \& Witt, A. N. 1967, AJ, 72, 1334

Duquennoy, A., \& Mayor, M. 1991, A\&A, 248, 485

Eaton, J. A., \& Williamson, M. H. 2004, SPIE, 5496, 710

Eaton, J. A., \& Williamson, M. H. 2007, PASP, 119, 886

Edvardssen, B., Andersen, J., Gustafsson, B., et al. 1993, A\&A, 275, 101

Fekel, F. C. 1997, PASP, 109, 514

Fekel, F. C., Boden, A. F., Tomkin, J., \& Torres, G. 2009a, ApJ, 695, 1527

Fekel, F. C., Rajabi, S., Muterspaugh, M. W., \& Williamson, M. H. 2013a, AJ, 145,111

Fekel, F. C., \& Tomkin, J. 2004, AN, 325, 649

Fekel, F. C., Tomkin, J., \& Williamson, M. H. 2009b, AJ, 137, 3900

Fekel, F. C., Tomkin, J., \& Williamson, M. H. 2013b, AJ, 146, 129

Flower, P. J. 1996, ApJ, 469, 355

Girardi, L., Bressan, A., Bertelli, G., \& Chiosi, C. 2000, A\&AS, 141, 371

Goldberg, D., Mazeh, T., Latham, D. W., Stefanik, R. T., Carney, B. W., \& Laird, J. B. 2002, AJ, 124, 1132

Gomez, A. E., \& Abt, H. A. 1982, PASP, 94, 650

Gray, D. F. 1992, The Observation and Analysis of Stellar Photospheres (Cambridge: Cambridge Univ. Press)

Gray, R. O., Corbally, C. J., Garrison, R. F., McFadden, M. T., \& Robinson, P. E. 2003, AJ, 126, 2048

Griffin, R. F. 2013, AJ, 146, 13

Halbwachs, J. L. 1981, A\&AS, 44, 47

Halbwachs, J. L., Mayor, M., Udry, S., \& Arenou, F. 2003, A\&A, 397, 159

Hall, J. C., Lockwood, G. W., \& Skiff, B. A. 2007, AJ, 133, 862

Harlan, E. A. 1969, AJ, 74, 916

Hoffleit, D., \& Jaschek, C. 1982, The Bright Star Catalogue (4th ed.; New Haven, CT: Yale Univ. Obs.)

Holmberg, J., Nordström, B., \& Andersen, J. 2009, A\&A, 501, 941

Huenemoerder, D. P., \& Barden, S. C. 1984, BAAS, 16, 510

Hummel, C. A., Carquillat, J.-M., Ginestet, N., et al. 2001, AJ, 121, 1623

Hut, P. 1981, A\&A, 99, 126

Iliev, I. K., Fenovcik, M., Budaj, J., et al. 2004, in IAU Symp. 224, The A-Star Puzzle, ed. J. Zverko, J. Ziznovsky, S. J. Adelman, \& W. W. Weiss (Cambridge: Cambridge Univ. Press), 301

Jancart, S., Jorissen, A., Babusiaux, C., \& Pourbaix, D. 2005, A\&A, 442, 365 
Johnson, H. L., \& Morgan, W. W. 1953, ApJ, 117, 313

Kang, W., Lee, S. G., \& Kim, K. M. 2011, ApJ, 736, 87

Katoh, N., Itoh, Y., Toyota, E., \& Sato, B. 2013, AJ, 145, 41

Keenan, P. C., \& McNeil, R. C. 1989, ApJS, 71, 345

Lucy, L. B., \& Sweeney, M. A. 1971, AJ, 76, 544

Luyten, W. J. 1936, ApJ, 84, 85

Martinez-Arnaiz, R., Maldonado, J., Montes, D., Eiroa, C., \& Montesinos, B. 2010, A\&A, 520, A79

Massarotti, A., Latham, D. W., Stefanik, R. P., \& Fogel, J. 2008, AJ, 135, 209

Mazeh, T., Prato, L., Simon, M., et al. 2002, ApJ, 564, 1007

Mazeh, T., Simon, M., Prato, L., Markus, B., \& Zucker, S. 2003, ApJ, 599, 1344

McAlister, H. A. 1976, PASP, 88, 317

McCarthy, J. A., Sandiford, B. A., Boyd, D., \& Booth, J. 1993, PASP, 105,881

Mishenina, T. V., Soubiran, C., Bienaymé, O., et al. 2008, A\&A, 489, 923

Nakajima, T., \& Morino, J. I. 2012, AJ, 143, 2

Nidever, D. L., Marcy, G. W., Butler, R. P., Fischer, D. A., \& Vogt, S. S. 2002, ApJS, 141, 503

Perryman, M. A. C., \& ESA 1997, The Hipparcos and Tycho Catalogues (Noordwijk: ESA)

Plaskett, J. S., Harper, W. E., Young, R. K., \& Plaskett, H. H. 1920, PDAO, 1,163
Pourbaix, D., \& Arenou, F. 2001, A\&A, 372, 935

Pourbaix, D., \& Boffin, H. 2003, A\&A, 398, 1163

Quirrenbach, A. 2001, ARA\&A, 39, 353

Sanford, R. F. 1922, ApJ, 56, 446

Scarfe, C. D., Batten, A. H., \& Fletcher, J. M. 1990, PDAO, 18, 21

Schröder, C., Reiners, A., \& Schmitt, J. H. M. M. 2009, A\&A, 493, 1099

Stockton, R. A., \& Fekel, F. C. 1992, MNRAS, 256, 575

Strassmeier, K. G., \& Fekel, F. C. 1990, A\&A, 230, 389

Strassmeier, K. G., Washuettl, A., Granzer, T., Scheck, M., \& Weber, M. 2000 A\&AS, 142, 275

Takeda, Y., Ohkubo, M., Sato, B., Kambe, E., \& Sadakane, K. 2005, PASJ, 57,27

Tassoul, J.-L., \& Tassoul, M. 1992, ApJ, 395, 259

Tassoul, J.-L., \& Tassoul, M. 1996, FCPh, 16, 377

Taylor, B. J. 2005, ApJS, 161, 444

Tomkin, J., \& Fekel, F. C. 2006, AJ, 131, 2652

Torres, G., Andersen, J., \& Giménez, A. 2010, A\&AR, 18, 67

van Leeuwen, F. 2007, Hipparcos, The New Reduction of the Raw Data (Dordrecht: Springer)

Wilson, O. C., \& Skumanich, A. 1964, ApJ, 140, 1401

Young, R. K. 1923, PDAO, 2, 205

Young, A., \& Koniges, A. 1977, ApJ, 211, 836

Zahn, J.-P. 1977, A\&A, 57, 383 